\title{
Determination of resistance pattern of bacteriostatic antimicrobial drugs by analysis of blood culture reports
}

\author{
Ashish Bhaskarrao Lawankar*, Momin M. Abdul Mujeeb, Rajesh Sudhakar Hiray
}

Department of Pharmacology, B. J. Government medical college, Pune, Maharashtra. India

\author{
Received: 10 December 2021 \\ Revised: 08 January 2022 \\ Accepted: 10 January 2022 \\ *Correspondence: \\ Dr. Ashish Bhaskarrao Lawankar, \\ Email: alawankar10@gmail.com
}

Copyright: (C) the author(s), publisher and licensee Medip Academy. This is an open-access article distributed under the terms of the Creative Commons Attribution Non-Commercial License, which permits unrestricted non-commercial use, distribution, and reproduction in any medium, provided the original work is properly cited.

\begin{abstract}
Background: Determination of each isolated bacterium from blood culture and pattern of antimicrobial sensitivity has an important role epidemiologically in a region and can assist physicians in the determination of primary antimicrobial agents. In this study, schema of resistance of bacteriostatic antimicrobials in blood culture is evaluated. Determination of resistance pattern of bacteriostatic antimicrobial drugs by analysis of blood culture report.

Methods: A retrospective analysis of microbiological blood culture and antibiotics sensitivity results have been done. A total of 120 laboratory culture results were selected randomly during the observational period. These all-blood culture reports were analyzed for bacteriostatic antimicrobial resistance pattern.

Results: The 41 bacterial isolates were obtained from 120 blood culture reports, $24.39 \%$ (10) were gram positive and $75.60 \%$ (31) were gram-negative. The most frequently isolated gram-negative organisms were K. pneumoniae (34.14\%) followed by Pseudomonas $(12.19 \%)$, E. coli $(12.19 \%)$. The most frequently isolated gram-positive organism was staphylococcus aureus. All isolates showed less resistance to bacteriostatic antibiotics like clindamycin, chloramphenicol, co-trimoxazole, doxycycline, linezolid, tetracycline, nitrofurantoin, erythromycin, tigecycline.

Conclusions: S. aureus, E. coli and Klebsiella spp. pseudomonas aeruginosa were the leading causes of septicaemia or any type of infection in our study finding. These bacteria isolates were highly resistant to bactericidal antimicrobials. But bacteriostatic antimicrobial has shown less resistance compared to bactericidal.
\end{abstract}

Keywords: Bacteriostatic antimicrobials, Blood culture, Resistance pattern

\section{INTRODUCTION}

Septicaemia is very common emergency in medicine. It is due to various dangerous and lethal infection. These infections are mostly of bacterial, viral or parasitic origin. In such septicaemic infection, blood culture and other cultures should be done before antimicrobial therapy. Determination of each isolated bacterium or microorganisms from blood culture and pattern of antimicrobial sensitivity has an important role in a region. This can assist physicians in the determination of primary antimicrobial agents. ${ }^{1}$ In this study, schema of resistance of bacteriostatic antimicrobials in blood culture is the evaluated.

The WHO defines antimicrobial resistance (AMR) as resistance to an antimicrobial drug that was once able to treat an infection by that microorganism. AMR is now becoming a global problem to the public health worldwide. The world health organization (WHO) has declared AMR as public health concern and has urged different countries to develop action plan to combat the problem. Knowledge of healthcare associated infections (HCAIs) and the AMR patterns of the isolates are very important towards guiding 
empirical treatment. These data are crucial in rationalizing empirical treatment and set measures for surveillance. These can change policy in developing countries. ${ }^{2}$

Antimicrobial use is growing worldwide, and resistance to it is also increasing. Its impact is more in developing countries where infections are high. It is because of poverty, unhygienic environment, and poor health care facilities. In such scenario, the morbidity and mortality rate are also having increasing trend due to infectious diseases. The challenge of resistance also persists in developed countries. There is also increased probability of multidrug resistant organisms which have made the situation worsed. ${ }^{3}$

For more than 50 years, there are different alterations taken place in use of antimicrobials, such as change in kind and group of antimicrobials, sensitivity, and resistance pattern to various antibiotics. These changes are due to different causes such as emerging and re-emerging infectious disorders, over-the-counter use, and non-prescription utility of many antibiotics. Physicians and policy makers of every country can be helped by knowledge of trend in sensitivity and resistance patterns to make better decisions on their approach to resolve probable resistance.

Lack of national antibiotic resistance monitoring plan in developing countries will lead to unsuitable use in people and healthcare system workers. Development of infections and growing antibiotic resistance is a major concern around the world, and resistance pattern can be different according to geographic and epidemiological features. Sometimes these infections lead to multidrug resistance which can result in long-term hospitalization of patients, increase the rate of mortality, risk of other infections, and increased cost of the healthcare system. Therefore, in this investigation, the researchers analysed the pattern of AMR for different organisms at tertiary care hospital and teaching institute, Pune during Jan 2021-June2021, which can be a beneficial model for both clinicians in empirical therapy and policy makers.

\section{Aim and objectives}

The aim and objectives of the study were to determination of resistance pattern of bacteriostatic antimicrobial drugs by analysis of blood culture report.

\section{METHODS}

This is a descriptive observational study. It was performed on blood cultures requested at microbiology department, of B. J. GMC, Pune. This hospital is an academic medical institute where different types of disorders and infection can be treated. That's why this institute is a good source of information of status in AMR. A retrospective analysis of microbiological culture and antibiotics sensitivity results have been done. A total of 120 laboratory culture results were collected during the observational period.
Bloodstream infections (BSI) and sepsis are major causes of morbidity and mortality worldwide. Blood culturebased diagnostics usually requires 1-2 days for identification of bacterial agent and an additional 2-3 days for phenotypic determination of antimicrobial susceptibility pattern. With the escalating burden of AMR rapid diagnostics becomes increasingly important to secure adequate antibiotic therapy. Results from this study hold great promise for future applications in clinical microbiology and for health care surveillance purposes.

\section{RESULTS}

In the period of study, reports of 120 blood cultures were collected from microbiology laboratories. The prevalence of positive cultures growth was 41. The frequency of bacteria isolated from blood cultures of patients is shown in Figure 1 and Table 1.

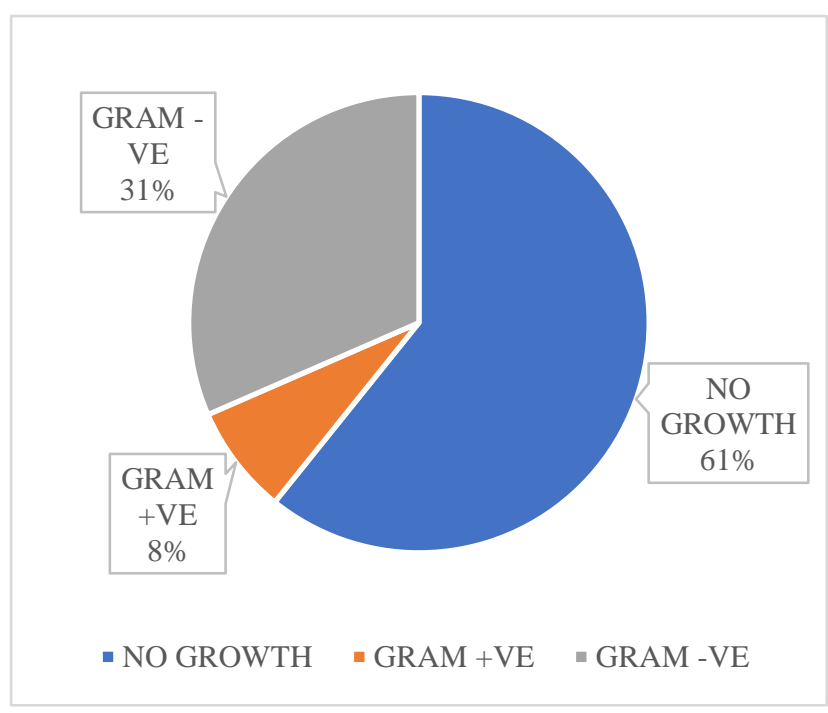

Figure 1: Culture growth reports analysis.

Table 1: Percentage of bacterial growth isolated from blood culture.

\begin{tabular}{|l|l|}
\hline Bacterial growth isolated & Percentage (\%) \\
\hline Klebsiella pneumonia & 34.14 \\
\hline Pseudomonas aeruginosa & 12.19 \\
\hline E. coli & 12.19 \\
\hline Non-fermented gram-ve bacilli & 7.31 \\
\hline Acinetobacter & 2.43 \\
\hline Citrobacter Kosai & 2.43 \\
\hline Serratia species & 2.43 \\
\hline Enterobacter species & 2.43 \\
\hline Staphylococcus aureus & 24.39 \\
\hline
\end{tabular}

Out of 41 bacterial isolates, $24.39 \%$ (10) were gram positive and $75.60 \%$ (31) were gram-negative. The most frequently isolated gram-negative organisms were $K$. pneumoniae (34.14\%) followed by Pseudomonas (12.19\%), E. coli (12.19\%), Acinetobacter, Citrobacter Kosai, Serratia species, non-fermented gram -ve bacilli and Enterobacter species (Figure 1 and Table 1). The most 
frequently isolated gram-positive organism was Staphylococcus aureus. All isolates showed less resistance to bacteriostatic antibiotics like clindamycin, chloramphenicol, co-trimoxazole, doxycycline, linezolid, tetracycline, nitrofurantoin, erythromycin, tigecycline. Resistance pattern to these drugs is given in the Table 2.

Table 2: Resistance and sensitivity pattern observed.

\begin{tabular}{|lll|}
\hline $\begin{array}{l}\text { Antimicrobial } \\
\text { drugs }\end{array}$ & $\begin{array}{l}\text { Resistance } \\
(\%)\end{array}$ & $\begin{array}{l}\text { Sensitivity } \\
(\%)\end{array}$ \\
\hline Clindamycin & 0 & 100 \\
\hline Chloramphenicol & 22.22 & 77.78 \\
\hline Co-trimoxazole & 45.45 & 54.55 \\
\hline Doxycycline & 0 & 100 \\
\hline Erythromycin & 20 & 80 \\
\hline Linezolid & 0 & 100 \\
\hline Nitrofurantoin & 33.33 & 66.66 \\
\hline Tigecycline & 0 & 100 \\
\hline
\end{tabular}

In our study, all the isolates were resistant to bactericidal drugs Ampicillin, amoxicillin, cefixime, ceftizoxime, norfloxacin. Augmentin with clavulanic acid, aztreonam, cefepime, ceftazidime, ceftriaxone penicillin had the lowest sensitivity to all bacterial isolates. Whereas other bactericidal drugs like amikacin, cefoperazone, cefotaxime, cefoxitin, cefuroxime, ciprofloxacin, imipenem, piperacillin-tazobactam had moderate sensitivity. Carbenicillin, colistin, gentamycin, levofloxacin, ofloxacin, teicoplanin, vancomycin had the highest sensitivity.

In bacteriostatic drugs, clindamycin, doxycycline, linezolid, tigecycline are highly sensitive to Citrobacter Kosai, E. coli, Pseudomonas aeruginosa, Klebsiella pneumonia and Staphylococcus aureus. Antibiotics resistance pattern of another bacteriostatic antimicrobial is given in Table 3.

Table 3: Comparison of resistance pattern of bacteriostatic drugs.

\begin{tabular}{|llll|l|}
\hline Growth/ antibiotics & $\begin{array}{l}\text { Chloramphenicol } \\
(\%)\end{array}$ & $\begin{array}{l}\text { Co-trimoxazole } \\
(\%)\end{array}$ & $\begin{array}{l}\text { Erythromycin } \\
(\%)\end{array}$ & $\begin{array}{l}\text { Nitrofurantoin } \\
(\%)\end{array}$ \\
\hline Klebsiella pneumonia & 33.33 & 44.44 & - & 33.33 \\
\hline Pseudomonas aeruginosa & 0 & 0 & - & - \\
\hline E. coli & 0 & 50 & - & 50 \\
\hline Non-fermented gram -ve bacilli & - & 50 & - & - \\
\hline Acinetobacter & 0 & 100 & - & - \\
\hline Citrobacter Kosai & - & - & - & - \\
\hline Serratia species & - & 0 & - & - \\
\hline Enterobacter species & 100 & 100 & 20 & - \\
\hline Staphylococcus aureus & - & 40 & & - \\
\hline
\end{tabular}

\section{DISCUSSION}

AMR is a major clinical problem in treating infections caused by microorganisms. The resistance to the antimicrobials has increased over the years. Resistance rates vary from country to country and region to region. Overall, isolates from Latin American countries show the high resistance rates to all antimicrobial agents followed by Asian-Pacific isolates and European strains; while the strains from Canada exhibit the lowest resistance pattern by Gales et al. ${ }^{4}$

In this study, it was accounted that majority of the organisms isolated were the gram-ve. In which Klebsiella pneumonia was the predominant pathogen isolated. Klebsiella pneumonia has shown a slow but steady increase in resistance to several bacteriostatic antimicrobials like chloramphenicol, co-trimoxazole, nitrofurantoin. These drugs should no longer be prescribed as initial empirical therapy in our region.

Pseudomonas aeruginosa has shown high sensitivity to several bacteriostatic antimicrobials like chloramphenicol, co-trimoxazole. These drugs can be prescribed as initial empirical therapy in our region. E. coli has shown high sensitivity to bacteriostatic antimicrobials like chloramphenicol, but increased resistance to cotrimoxazole and nitrofurantoin. Hence chloramphenicol can be prescribed as initial empirical therapy in E. coli infection. Non-fermented gram-ve bacilli, Acinetobacter has shown increased resistance to co-trimoxazole. Enterobacter species has shown almost complete resistance to chloramphenicol and co-trimoxazole.

Gram + ve bacterial isolates like staphylococcus aureus has shown increasing resistance to several bacteriostatic antimicrobials like co-trimoxazole, erythromycin. These drugs should not be prescribed as initial empirical therapy in our region.

As noted from other studies, S. aureus and Klebsiella pneumonia were the most common bacterial isolates from blood culture. However, their sources remain unknown from the present study though other studies have documented both endogenous and exogenous sources from hospital environment could be potential one by Seni et al. ${ }^{16}$

Most of the blood culture tests were colonized with a single bacterial species. These data are confirmed by Mohammed et al that found single bacterial growth, while 
Yeong et al showed a higher number of polymicrobial resistant species in wound bacterial cultures within the first 72 hour. $^{17,18}$

We found that a substantial proportion of pathogens isolated from blood culture demonstrated AMR, principally among methicillin-resistant $S$. aureus and gram-negative organisms. These pathogens were commonly resistant to first line antibiotic drugs (e.g., fluoroquinolones, penicillin's, ceftriaxone) at rates that were much higher than those reported with bacteriostatic anti-microbial drugs. Similar surveillance has been seen in other countries in Africa, such as Kenya. In general, the most common aetiologies of bacteraemia here, as well as in the United States and several countries in Europe, were similar. The exception was gram-ve microorganism, which was isolated more frequently in India and African countries where low socioeconomic strata are more.

\section{Limitations}

The analysis based on getting information from microbiological request/report forms therefore it was difficult to tell whether the infection originated from the community, or it was healthcare-associated. Other important epidemiological information such as patient's outcome, duration of hospital stays etc. was not reported. The limitations of this study are the small sample size and being a retrospective study, it was difficult to retrieve all the relevant clinical data which could have given more inputs regarding other contributing factors for the development of resistance pattern of bacteriostatic antimicrobial drugs.

\section{CONCLUSION}

S. aureus, E. coli and Klebsiella spp. Pseudomonas aeruginosa were the leading causes of septicaemia or any type of infection in our study finding. These bacteria isolates were highly resistant to bactericidal antimicrobials. But bacteriostatic antimicrobial has shown less resistance compared to bactericidal. High utilization rate of antibiotics is the most important contributory factor for the development of AMR and continuous surveillance is needed in order to keep national guidelines on antimicrobial therapy updated. More studies are needed in view of AMR pattern to bacteriostatic and bactericidal drugs.

\section{Funding: No funding sources}

Conflict of interest: None declared

Ethical approval: The study was approved by the Institutional Ethics Committee

\section{REFERENCES}

1. Keihanian F, Saeidinia A. Epidemiology of antibiotic resistance of blood culture in educational hospitals in Rasht, North of Iran. Infection Drug Resistance. 2018;11:1723-8.
2. Moremi N, Claus H, Mshana SE. Antimicrobial resistance pattern: a report of microbiological cultures at a tertiary hospital in Tanzania. BMC Infect Dis. 2016;16:756.

3. Muhammad N, Adil M, Naz SM, Abbas SH, Khan MZUI et al. Resistance and sensitivity pattern of Staphylococcus Aureus; A study in Lady Reading Hospital Peshawar. J Postgrad Med Inst. 2013;27(1):42-7.

4. Rahman F, Chowdhury S. Antimicrobial Resistance Pattern of Gram-negative Bacteria Causing Urinary Tract Infection. Stamford J Pharmaceutical Sci. 2013;2(1):44-50.

5. Shruthi KS, Sanji N, Raghoji CR, Guruprasad G, Shashikala GH. A retrospective study on antibiotic sensitivity pattern of pathogens isolated from blood culture in cases of late-onset neonatal sepsis at a neonatal intensive care unit. Natl J Physiol Pharm Pharmacol. 2018;8(5):709-12.

6. Basri DF, Xian LW. Bacteriostatic Antimicrobial Combination: Antagonistic Interaction between Epsilon-Viniferin and Vancomycin against Methicillin-Resistant Staphylococcus aureus. BioMed Res Int Vol. 2014;461756:8.

7. Kaur DC, Chate SS. Study of antibiotic resistance pattern in methicillin resistant Staphylococcus Aureus with special reference to newer antibiotic. J Global Infect Dis. 2015;7:78-84.

8. Sorsa A, Früh J. Blood culture result profile and antimicrobial resistance pattern: a report from neonatal intensive care unit (NICU), Asella teaching and referral hospital, Asella, southeast Ethiopia. Antimicrobial Resistance Infect Control. 2019;8:42.

9. De Kraker M, Jarlier V, Monen J, Heuer O, Van De Sande N, Grundmann H. The changing epidemiology of bacteraemias in Europe: trends from the European Antimicrobial Resistance Surveillance System. Clin Microbiol Infect. 2013;19(9):860-8.

10. Saeidynia F, Keihanian F, Saeidynia A. Antibiotic resistance in blood culture samples from patients referred to Razi laboratory of Rasht, 2006-2011. Adv Infect Dis. 2014;04(03):165-72.

11. Orsi GB, Falcone M, Venditti M. Surveillance, and management of multidrug-resistant microorganisms. Expert Rev Anti Infect Ther. 2011;9:653-79.

12. Barrett SP, Savage MA, Rebec MP, Guyot A, Andrews N, Shrimpton SB. Antibiotic sensitivity of bacteria associated with community-acquired urinary tract infection in Britain. J. Antimicrob. Chemother. 1999;44:359-65.

13. Rice LB. Federal funding for the study of antimicrobial resistance in nosocomial pathogens: No ESKAPE. J Infect Dis. 2008;197:1079-81.

14. Udobi CE, Obajuluwa AF, Onaolapo JA. Prevalence and antibiotic resistance pattern of methicillinresistant Staphylococcus aureus from an orthopaedic hospital in Nigeria. BioMed Res Int Vol. 2013;860467:4.

15. Debasmita D, Shakti R, Mahesh CS, Lolly P, Debata NK, Rabindra NP. Surveillance of infection status of 
drug resistant Staphylococcus aureus in an Indian teaching hospital. Asian Pac J Trop Dis. 2013;3:13342.

16. Seni J, Najjuka CF, Kateete DP, Makobore P, Joloba MP, Kajumbula MP et al. Antimicrobial resistance in hospitalized surgical patients: a silently emerging public health concern in Uganda. BMC Research Notes. 2013;6:298.

17. Mohammed A, Seid ME, Gebrecherkos T, Tiruneh M, Moges F. Bacterial Isolates and Their Antimicrobial Susceptibility Patterns of Wound Infections among Inpatients and Outpatients Attending the University of Gondar Referral Hospital, Northwest Ethiopia. Int J Microbiol. 2017;8953829.

18. Yeong EK, Sheng WH, Hsueh PR, Hsieh SM, Huang HF, Ko AT et al. The Wound Microbiology and the Outcomes of the Systemic Antibiotic Prophylaxis in a
Mass Burn Casualty Incident. J. Burn Care Res. 2020;41:95-103.

19. Puca V, Marulli RZ, Grande R, Vitale I, Niro A, Molinaro $G$ et al. Microbial Species Isolated from Infected Wounds and Antimicrobial Resistance Analysis: Data Emerging from a Three-Years Retrospective Study. Antibiotics. 2021;10:1162.

20. Kajumbula H, Fujita AW, Mbabazi O. Antimicrobial Drug Resistance in Blood Culture Isolates at a Tertiary Hospital, Uganda. Emerg Infect Dis. 2018;24(1):174-5.

Cite this article as: Lawankar AB, Mujeeb MMA, Hiray RS. Determination of resistance pattern of bacteriostatic antimicrobial drugs by analysis of blood culture reports. Int J Basic Clin Pharmacol 2022;11:117-21. 